\title{
Pandemic Makes Chronic Disease Prevention a Priority
}

\author{
Karen S. Kmetik, PhD, Alexis Skoufalos, EdD, ${ }^{2}$ and David B. Nash, MD, MBA ${ }^{2}$
}

Keywords: COVID-19, pandemic, chronic disease, diabetes, prevention

T HE COVID-19 PANDEMIC has shone a spotlight on the growing chronic disease crisis in America. Available data suggest that underlying chronic disease, including cardiovascular disease and diabetes, among other factors, may put individuals at higher risk for complications and a more severe course of illness if they contract COVID-19. ${ }^{1}$ In 2017 alone, pre-pandemic, cardiovascular-related deaths totaled nearly 860,000 in the United States, and many of those deaths may have been preventable. ${ }^{2}$ There are approximately 34.1 million Americans with diabetes, and another 88 million adults have prediabetes, ${ }^{3}$ a precursor to diabetes that can potentially be reversed.

The COVID-19 pandemic has heightened our awareness of the critical need to prevent the incidence of both infectious disease and chronic disease. The health care community will require a renewed mindset to make prevention of chronic disease for all people a top priority. We will need to begin working together across many sectors like never before to better serve individuals, communities, physicians, and care teams. If we act now and seize this moment, we can improve population health significantly.

Efforts are already underway to build the infrastructure to support such a sea change in health care. Over the past 5 years, the American Medical Association (AMA) and the Centers for Disease Control and Prevention (CDC) have joined with numerous collaborators to bring awareness and education to communities across the country about prediabetes, its associated consequences, and steps individuals and clinicians can take to potentially reverse the condition and prevent the occurrence of diabetes.

The goal of these efforts is to ensure that every American adult knows their risk for diabetes; that physicians and care teams are identifying patients with prediabetes and referring eligible patients to an evidenced-based National Diabetes Prevention Program (DPP) lifestyle change program; and that programs are covered regardless of the individual's health plan (public or private). We also want to see entities that provide the National DPP lifestyle change program supported to use modalities that meet the needs of people where they are (including online and distance learning options). Additionally, we aim to ensure that all communities are better equipped to serve their populations by offering equal access to resources and solutions that support chronic disease prevention and healthier lifestyles.

One such shining example of this work is underway in Philadelphia where the Jefferson College of Population Health, with support from the AMA and CDC, is serving as the backbone organization for the Philadelphia Diabetes Prevention Collaborative (PDPC). The PDPC regional initiative is focused on diabetes prevention in the 5-county area known as Greater Philadelphia. Philadelphia is the poorest and most diverse of the nation's 10 largest cities ${ }^{4}$ and ranks at the bottom of the state's 67 counties in terms of health outcomes. However, it also is home to a strong network of community-based organizations, a health information exchange, 5 academic health systems, and a large employer coalition working on disease prevention and health promotion initiatives. Representatives from these key stakeholders and other groups began working together in 2019 toward a goal of " 2000 in 2020" - enrolling 2000 individuals into a National DPP lifestyle change program in 2020.

The collaborative is putting their collective efforts into diabetes prevention in an area of the country with enormous health disparities, each contributing expertise and resources to raise awareness among individuals and clinicians; increase access to in-person, distance learning, and online National DPP lifestyle change programs; and build capacity of organizations to deliver the program. Working groups are now at work creating an online resource that will direct and refer people to a conveniently located National DPP lifestyle change program, providing training to lifestyle coaches to deliver the CDC-approved curriculum, increasing the availability of National DPP lifestyle change programs offered in multiple languages in order to reach traditionally underserved populations, supporting community-based organizations to offer the program, and advocating for expanded coverage from health plans and employers.

The COVID-19 pandemic has provided health care organizations and health care providers the opportunity to not only rethink the delivery of health care, but also to prioritize

\footnotetext{
${ }^{1}$ American Medical Association, Chicago, Illinois, USA.

${ }^{2}$ Jefferson College of Population Health, Philadelphia, Pennsylvania, USA.
} 
the prevention of chronic disease. In Philadelphia, Jefferson's Center for Urban Health took the opportunity to build upon their successful 2018 pilot using a video conferencing platform to deliver the National DPP lifestyle change program. Neva White, Senior Health Educator of the Center, found that the distance learning program resulted in greater engagement and retention, and more people reaching their weight loss and physical activity goals. The latest cohort began in February 2020 with 32 participants, all of whom remain actively engaged in the program.

Nationwide, other innovative health care leaders have quickly pivoted to use technology platforms to support and expand their diabetes prevention strategies in order to accommodate social distancing practices. For example:

- Henry Ford Health System provided diabetes prevention coaches with the instruction and tools needed to start a distance learning model for delivering the CDCapproved curriculum. Distance learning removed barriers for some participants. It also afforded opportunities for the coaches and staff to connect one-on-one with participants and provide resources to address their social needs.

- UCLA and other University of California campuses that had been offering the National DPP lifestyle change program to employees, patients, and students, strengthened their resolve to enhance diabetes and obesity prevention efforts as a result of the pandemic. The UCLA DPP transitioned to virtual delivery and has found that this provides a sense of continuity and stability for DPP participants during this difficult time.

- Intermountain Healthcare adjusted its diabetes prevention curriculums by facilitating its Intermountain Diabetes Prevention program (ie, Prediabetes 101, Medical Nutrition Therapy, Weigh to Health) using video visits and web-based group curriculum. The organization is also working with its primary care teams and population health management organizations to identify clinical staff members who can conduct outreach to patients with prediabetes to enroll them in program pathways that are delivered virtually and through distance learning opportunities.

The COVID-19 pandemic heralds a sea change for health care and the realities we must face are clear. ${ }^{5}$ The likelihood of a second surge is high; to avoid an even higher toll, we must take steps to ensure that our population is in better health the next time. We have no more time to waste, and no excuses are worth the suffering and loss. As physicians, care team members, and health care leaders, we all must play a role in the relentless dedication to prevent chronic disease, such as diabetes and cardiovascular disease, and to ensure access to programs and healthy environments for all populations.

\section{Author Disclosure Statement}

Dr Kmetik is employed by the American Medical Association (AMA), which supported the Jefferson College of Population Health (JCPH) to serve as the backbone organization for the Philadelphia Diabetes Prevention Collaborative. Drs Skoufalos and Nash are employed by JCPH. The authors retain sole responsibility for the content of this paper. The views expressed herein are those of the authors and should not be construed as the official positions of the AMA or JCPH.

\section{Funding Information}

The American Medical Association supported the Jefferson College of Population Health $(\mathrm{JCPH})$ through a grant to serve as the backbone organization for the Philadelphia Diabetes Prevention Collaborative.

\section{References}

1. CDC COVID-19 Response Team. Preliminary estimates of the prevalence of selected underlying health conditions among patients with coronavirus disease 2019-United States, February 12-March 28, 2020. MMWR Morb Mortal Wkly Rep 2020;69:382-386.

2. Virani SS, Alonso A, Benjamin EJ, et al. Heart disease and stroke statistics-2020 update: a report from the American Heart Association. Circulation 2020;141:e139-e596.

3. Centers for Disease Control and Prevention. National Diabetes Statistics Report 2020: Estimates of Diabetes and Its Burden in the United States. www.cdc.gov/diabetes/pdfs/ data/statistics/national-diabetes-statistics-report.pdf Accessed May 14, 2020.

4. McPhillips D. How racially and ethnically diverse is your city? www.usnews.com/news/cities/articles/2020-01-22/mea suring-racial-and-ethnic-diversity-in-americas-cities Accessed May 19, 2020.

5. Berwick DM. Choices for the "new normal." JAMA. Published online ahead of print May 4, 2020. DOI: 10.1001/jama.2020.6949.

Address correspondence to: Alexis Skoufalos, EdD

Jefferson College of Population Health 901 Walnut Street, 10th Floor Philadelphia, PA 19107

USA

E-mail: alexis.skoufalos@jefferson.edu 